\title{
Altered p-JAK1 expression is associated with estrogen receptor status in breast infiltrating ductal carcinoma
}

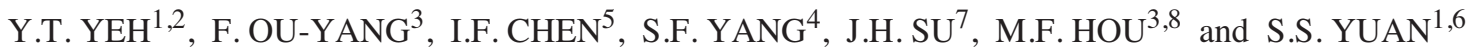 \\ ${ }^{1}$ Department of Medical Research and Department of Obstetrics and Gynecology, E-DA Hospital, Kaohsiung County; \\ ${ }^{2}$ Graduate Institute of Medicine, Departments of ${ }^{3}$ Surgery and ${ }^{4}$ Pathology, Kaohsiung Medical University, Kaohsiung; \\ Departments of ${ }^{5}$ Biomedical Engineering and ${ }^{6}$ Healthcare Administration, I-Shou University, Kaohsiung County; \\ ${ }^{7}$ Department of Obstetrics and Gynecology, Kaohsiung Medical University; ${ }^{8}$ National Sun Yat-Sen University- \\ Kaohsiung Medical University Joint Research Center, Kaohsiung, Taiwan, ROC
}

Received June 26, 2006; Accepted September 12, 2006

\begin{abstract}
The mammalian Janus kinase (JAK) family consists of four members, namely JAK1, JAK2, JAK3 and TYK2, which play a critical role in cytokine/growth factor signaling and is increasingly associated with human cancers. Aberrant activation of these non-receptor tyrosine kinases may contribute to carcinogenesis. Herein, we focused on exploring the potential role of $\mathrm{p}-\mathrm{JAK} 1$ in breast cancer. The expression profiles of p-JAK1 were analyzed in 68 pairs of cancer and non-cancer breast tissues from the same infiltrating ductal carcinoma case by using immunoblotting technique. The results obtained were further correlated with clinicopathological characteristics. Intriguingly, p-JAK1 expression was decreased in $55.9 \%$ of breast cancer tissues as compared to the matched noncancer tissues. Further immunohistochemistry study showed an intense p-JAK1 staining predominantly in adjacent normal breast tissues but not the matched cancer lesions. Decreased p-JAK1 expression in breast cancer tissues was significantly correlated with positive estrogen receptor (ER) status and increased tumor size ( $\mathrm{p}=0.010$ and 0.009 ). We also found that p-JAK1 expression was high in ER $\alpha$-negative breast cancer cell lines but was low in ER $\alpha$-positive breast cell lines. Transfection of ER $\alpha$-positive MCF-7 cells with an ER $\alpha$-specific siRNA upregulated the expression of p-JAK1. In summary, our results indicated that an altered p-JAK1 expression might be involved in the development of breast infiltrating ductal carcinoma in an ER $\alpha$-related manner.
\end{abstract}

\section{Introduction}

The mammalian Janus kinase (JAK) family consists of four members (JAK1, JAK2, JAK3 and TYK2), which are

Correspondence to: Dr S.S. Yuan, Department of Medical Research, E-DA Hospital, Kaohsiung County, Taiwan 824, ROC E-mail: yuanssf@ms33.hinet.net

Key words: breast cancer, infiltrating ductal carcinoma, p-JAK1, estrogen receptor associated with immune disorders and hematopoietic cancers (1-3). However, the potential role of JAK family in solid tumor, such as breast cancer, remains mostly unknown. It has been demonstrated that Jak1-/- mice died perinatally (4). Besides, constitutively activated JAK1 is observed in v-Src-transformed cells $(5,6)$. It appeared to implicate that JAK1 might play some role in tumorigenesis. However, controversially, there is evidence that Jak1-deficient cell lines were more tumorigenic than wild-type cells (7), while overexpression of BRCA1, a tumor suppressor gene closely associated with breast cancer, in non-cancerous epithelial EcR-293 cell line upregulates expression of JAK1 (8). The evidence indicates that JAK1 may work as a tumor suppressor or an oncogene under certain conditions or cell contents.

Different JAKs mediate specific biological functions, depending on the ligand or the ligand-specific receptor. For example, JAK2 mediates the growth effects of growth hormone (GH) and prolactin (PRL) on mammary epithelial cells $(9,10)$. Estrogen, which is closely involved in the development and carcinogenesis of breast tissues, inhibits GH signaling by suppressing GH-induced JAK2 phosphorylation (11). Although the information is limited, a recent study indicates that both JAK 1 and JAK2 are involved in the synthetic progestin medroxyprogesterone acetate (MPA) induced mammary adenocarcinomas in BALB/c mice and in the human breast cancer cell line T47D (12), indicating that the interplay between JAK signaling and hormone receptors may contribute to breast cancer development.

In this study, we analyzed the expression of p-JAK 1 in breast cancer tissues and adjacent non-cancer breast tissues from the same patients by immunoblotting and immunohistochemistry approaches. Herein, we show that p-JAK1 is aberrantly expressed in breast cancer tissues and may be involved in the development of breast cancer in an estrogen receptor related manner.

\section{Materials and methods}

Tissue collection and cell culture. Sixty-eight patients with breast infiltrating ductal carcinoma were included in this study (Table I) and the tissue specimens were obtained from the 
Table I. Clinicopathological characteristics of breast infiltrating ductal carcinoma cases (case no. 68).

\begin{tabular}{lll}
\hline Characteristics & $\mathrm{n}$ & $(\%)$ of patients \\
\hline
\end{tabular}

$\begin{array}{lrr}\text { Stage, } \mathrm{n} & & \\ \text { I } & 10 & 14.7 \\ \text { II } & 51 & 75.0 \\ \text { III } & 7 & 10.3\end{array}$

Grade, $\mathrm{n}$

$\begin{array}{lll}\text { I } & 19 & 27.9 \\ \text { II } & 22 & 32.4 \\ \text { III } & 27 & 39.7\end{array}$

Tumor size, $\mathrm{n}$

$\begin{array}{lll}\mathrm{T} 1(<2 \mathrm{~cm}) & 11 & 16.2 \\ \mathrm{~T} 2(2-5 \mathrm{~cm}) & 34 & 50.0 \\ \mathrm{~T} 3(>5 \mathrm{~cm}) & 23 & 33.8\end{array}$

$\mathrm{LN}$ metastasis, $\mathrm{n}$

Negative $\quad 33 \quad 48.5$

Positive $\quad 35 \quad 51.5$

Estrogen receptor status, $\mathrm{n}$

Negative $\quad 29 \quad 42.6$

Positive $\quad 39 \quad 57.4$

Progesterone receptor status, $\mathrm{n}$

$\begin{array}{lll}\text { Negative } & 44 & 64.7\end{array}$

Positive $24 \quad 35.3$

Her2/Neu status, $\mathrm{n}$

$\begin{array}{lll}\text { Negative } & 27 & 39.7 \\ \text { Positive } & 41 & 60.3\end{array}$

p-JAK1 status, $n$

\begin{tabular}{lrr}
$\mathrm{N}>\mathrm{C}$ & 38 & 55.9 \\
$\mathrm{~N}=\mathrm{C}$ & 5 & 7.4 \\
$\mathrm{~N}<\mathrm{C}$ & 25 & 36.8 \\
\hline
\end{tabular}

patients who underwent surgical treatment at the Department of Surgery, Kaohsiung Medical University Hospital, during the period from 2000 to 2003. The specimens were frozen immediately in liquid $\mathrm{N}_{2}$ and stored at $-80^{\circ} \mathrm{C}$ until further analysis. The ethics committee approved the study and informed consent was obtained from each patients. The clinicopathological characteristics of the patients were recorded. The histological types of the primary tumor were determined according to a system based on a modification of the WHO classification. The staging of the breast cancer was defined according to the TNM system. ER-positive breast cell lines (MCF-7, ZR75-1 and T47D) and ER-negative breast cancer cell lines (MDA-MB-231, SKBR-3 and MDA-MB-468) were kindly provided by Dr M.C. Hung at M.D. Anderson Cancer Center and were grown in DMEM/F12 medium supplemented with $10 \% \mathrm{FBS}, 100 \mathrm{U} / \mathrm{ml}$ penicillin, and $100 \mu \mathrm{g} / \mathrm{ml}$ streptomycin. Epithelial growth factor was from Sigma.
Immunoblot analysis. The frozen breast tissues were ground up and dissolved in EBC buffer (50 mM Tris pH 7.6, $120 \mathrm{mM}$ $\mathrm{NaCl}, 0.5 \%$ Nonidet P-40, $1 \mathrm{mM}$ EDTA, $1 \mathrm{mM}$ 2-mercaptoethanol, $50 \mathrm{mM} \mathrm{NaF}, 1 \mathrm{mM} \mathrm{Na} \mathrm{VO}_{4}$ ). MCF-7 breast cancer cells, treated with epidermal growth factor (EGF, $100 \mathrm{ng} / \mathrm{ml}$ ) in charcoal-stripped medium for $20 \mathrm{~min}$, were used as a positive control. The detailed procedures for immunoblotting were followed as described (13). Mouse monoclonal anti-p-JAK1, and rabbit polyclonal anti-JAK1 antibodies were from Santa Cruz. For the comparison of the expression levels of p-JAK1 in cancer versus non-cancer tissues, the AlphaImager ${ }^{\text {TM }} 1220$ documentation and analysis system was used (Alpha Innotech Corp.) (14). $\mathrm{N}>\mathrm{C}$ was defined as a $>50 \%$ higher expression of p-JAK1 in non-cancer tissue than in paired cancer tissue, after normalization by $\mathrm{B}$-actin (Fig. 1A). $\mathrm{N}<\mathrm{C}$ was defined as a $>50 \%$ higher expression of p-JAK 1 in cancer tissue than in paired non-cancer tissue, after normalization by $\mathrm{B}$-actin. $\mathrm{N}=\mathrm{C}$ was defined as a $<50 \%$ difference in p-JAK1 expression between the cancer tissue and the paired non-cancer tissue, after normalization by $\beta$-actin.

Immunohistochemistry. The detailed protocol for immunohistochemistry was as described previously (14). In brief, the tissue samples were fixed with $10 \%$ buffered formalin, then dissected, dehydrated and coated with wax. The samples were sliced to a thickness of $4 \mu \mathrm{m}$, and then either dyed with hematoxylin and eosin or immunostained with the first antibody (mouse monoclonal anti-estrogen receptor, anti-progesterone receptor and anti-Her2/Neu antibody from Dako; mouse monoclonal anti-p-JAK1, and rabbit polyclonal anti-JAK1 antibody from Santa Cruz), followed by Universal LAB+ kit/HRP (Dako) and counterstained with hematoxylin. The results were captured by a Nikon E-800M microscope, and then processed by Kodak MGDS330 and Adobe Photoshop 6.0.

Statistical analysis. The statistical analyses were performed using the SPSS 10.0 statistical package for PC (SPSS, Inc., Chicago, IL). Groups of patients with different p-JAK1 expression levels $(\mathrm{N}>\mathrm{C}, \mathrm{N}=\mathrm{C}, \mathrm{N}<\mathrm{C})$ were correlated with tumor stage, tumor grade, lymph node (LN) status, tumor size, ER status, PR status and Her2/Neu status, using Spearman's rho coefficient analysis and Fisher's exact test. $\mathrm{P} \leq 0.05$ was considered statistically significant.

Small interfering RNA (siRNA) transient transfection. The siRNA kit for knocking down ER message was obtained from Santa Cruz. The sequences for ER $\alpha$ specific and control siRNA were not released by the manufacturer. MCF-7 cells were grown in 6-well plates and were at $60 \%$ confluence when transfected with $100 \mathrm{nM}$ siRNA or control siRNA, by using Lipofectamine 2000 reagent (Invitrogen). The transfected cells were grown in DMEM supplemented with $10 \%$ FBS for $48 \mathrm{~h}$ and then harvested for immunoblot analysis.

\section{Results}

The expression pattern of p-JAK1 in breast infiltrating ductal carcinoma cases. The clinicopathological characteristics of breast infiltrating ductal carcinoma cases are shown in Table I. To investigate the role of activated JAK1/p-JAK 1 in the 
A.

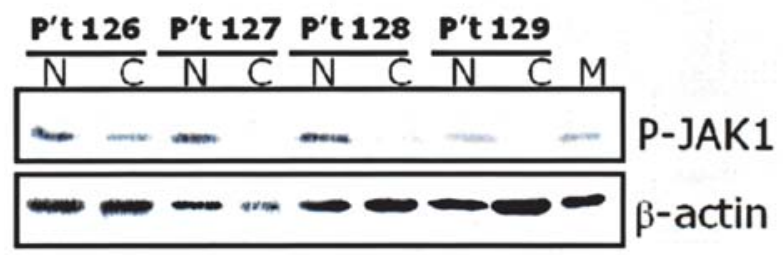

B.

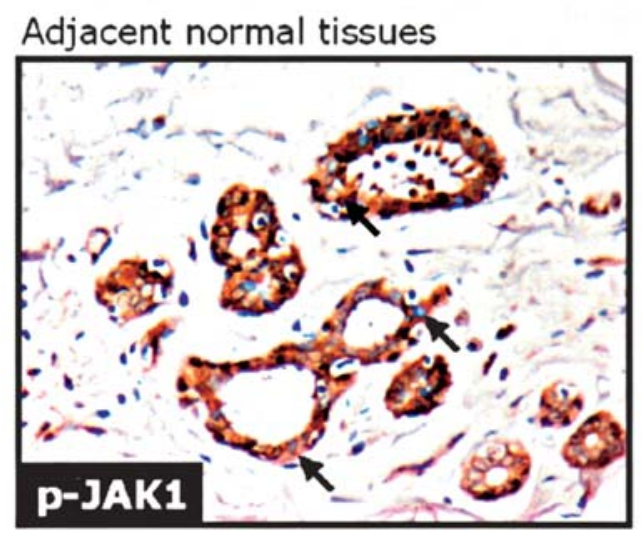

Breast cancer tissues

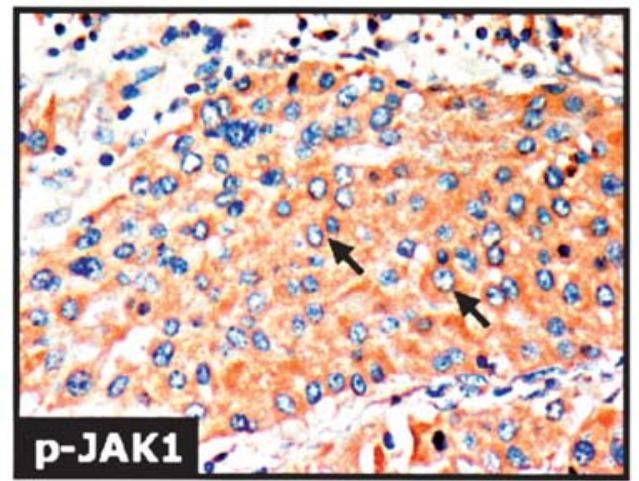

Figure 1. (A), Immunoblotting results show the expression patterns of p-JAK1 in breast cancer tissues (C) as compared to adjacent non-cancer breast tissues (N). MCF-7 cells, treated with $100 \mathrm{ng} / \mathrm{ml}$ EGF (epidermal growth factor), were included in last lane as the positive control (M). (B), Immunohistochemistry analysis showed the expression of p-JAK1 in breast cancer lesions and matched non-cancer breast tissues. Arrow indicates the cells positive for p-JAK immunostaining. A dramatically increased staining for p-JAK1 was observed in non-cancer breast tissues when compared to that in breast cancer lesions. Original magnification, $\mathrm{x} 200$.

carcinogenesis of breast infiltrating ductal carcinoma cases, immunoblot analysis was first applied to study the expression patterns of p-JAK 1 in the paired cancer and non-cancer tissues from the same patient (Fig. 1A). Interestingly, pJAK 1 expression was decreased in $55.9 \%$ of breast cancer tissues as compared to adjacent non-cancer breast tissues (Table I). Further immunohistochemistry analysis on the tissue section with a lower p-JAK1 expression in breast cancer tissues $(\mathrm{N}>\mathrm{C})$, determined by immunoblotting, also showed an intense p-JAK1 staining in non-cancer breast tissues, but not the matched cancer lesions (Fig. 1B).

The correlations between the expression patterns of p-JAK1 and the clinicopathological characteristics. The expression levels of p-JAK1 in breast cancer tissues were further correlated with tumor stage, tumor grade, LN status, tumor size, ER status, PR status and Her2/Neu status. Although the expression of p-JAK1 was decreased in $55.9 \%$ of the breast cancer tissues we analyzed (Table I), it was a surprise to note the positive correlation between the higher p-JAK1 expression in breast cancer tissues and the negative estrogen receptor (ER $\alpha)$ status as well as an increased tumor size $(\mathrm{p}=0.010$ and 0.009 , respectively) (Table II).

The expression of p-JAK1 in breast cancer cell lines was correlated with estrogen receptor status. The significant correlation between the expression of p-JAK1 and ER $\alpha$ in the

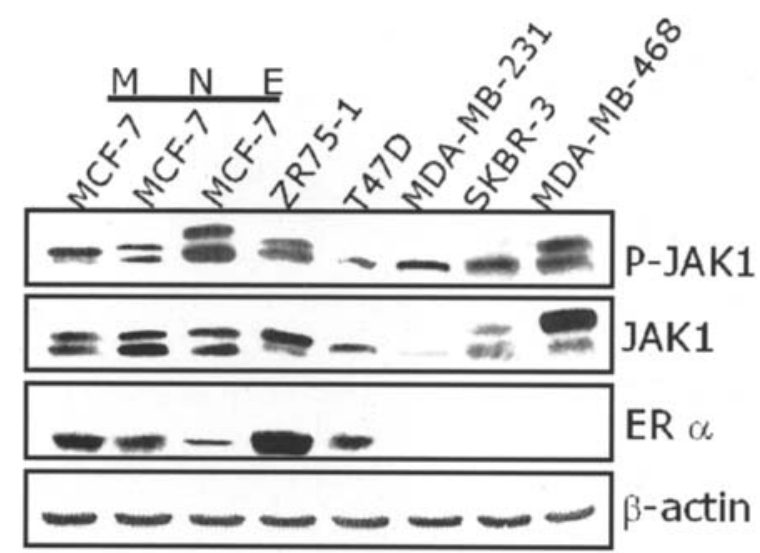

Figure 2. Immunoblot analysis showed the expression of p-JAK1 in the ERnegative breast cancer cell lines (MDA-MB-231, SKBR-3 and MDA-MB468) and ER-positive breast cell lines (MCF-7, ZR75-1 and T47D). The cells were cultured in a charcoal-stripped medium before harvest for immunoblotting analysis. E, transient transfection with ER $\alpha$ specific-siRNA; $\mathrm{N}$, transient transfection with control siRNA; M, mock transfection.

breast cancer tissues raised the question whether the expression of p-JAK1 was regulated by ER $\alpha$. To address this, different breast cancer cell lines with or without the expression of ER were analyzed by immunoblotting for their expression levels of p-JAK1 (Fig. 2). Surprisingly, all the 
Table II. Correlation of p-JAK1 expression with clinicopathological characteristics of breast infiltrating ductal carcinoma cases.

\begin{tabular}{|c|c|c|c|c|}
\hline Characteristics & $\mathrm{N}>\mathrm{C}$ & $\mathrm{N} \leq \mathrm{C}$ & $\mathrm{r}^{\mathrm{a}}$ & P-value \\
\hline Stage & & & -0.014 & 0.910 \\
\hline I & 4 & 6 & & \\
\hline II & 32 & 19 & & \\
\hline III & 2 & 5 & & \\
\hline Grade & & & -0.055 & 0.653 \\
\hline $\mathrm{I}$ & 11 & 8 & & \\
\hline II & 13 & 9 & & \\
\hline III & 14 & 13 & & \\
\hline Tumor size & & & 0.310 & 0.009 \\
\hline $\mathrm{T} 1$ & 3 & 8 & & \\
\hline $\mathrm{T} 2$ & 18 & 16 & & \\
\hline $\mathrm{T} 3$ & 17 & 6 & & \\
\hline LN metastasis & & & -0.092 & 0.454 \\
\hline Negative & 20 & 13 & & \\
\hline Positive & 18 & 17 & & \\
\hline ER status & & & 0.312 & 0.010 \\
\hline Negative & 11 & 18 & & \\
\hline Positive & 27 & 12 & & \\
\hline PR status & & & 0.036 & 0.768 \\
\hline Negative & 24 & 20 & & \\
\hline Positive & 14 & 10 & & \\
\hline Her2/Neu status & & & -0.055 & 0.655 \\
\hline Negative & 16 & 11 & & \\
\hline Positive & 22 & 19 & & \\
\hline
\end{tabular}

${ }^{\text {a}}$ Spearman's coefficient test.

ER-negative breast cancer cell lines we analyzed, including MDA-MB-231, SKBR-3 and MDA-MB-468, showed the higher p-JAK1 expression levels, in comparison to the ERpositive breast cell lines MCF-7, ZR75-1 and T47D (Fig. 2). Transient transfection of ER-specific siRNA also increased p-JAK1 expression in MCF-7 cells, as compared with the mock (no treatment) or control siRNA treatment. Neither the treatment of ER-specific siRNA nor the treatment of control siRNA affected JAK1 expression (Fig. 2).

\section{Discussion}

The expression of p-JAK1 was decreased in $55.9 \%$ of breast cancer tissues as compared to adjacent non-cancer breast tissues (Fig. 1 and Table I), implicating that the constitutive activation of JAK1 in non-cancer breast tissues might prevent tumorigenesis at least in certain breast cancer cases. It has been demonstrated that the induction of BRCA1 in non- cancerous epithelial EcR-293 cell line upregulates expression of JAK1 (2.4-fold) (8), and the enhanced expression of BRCA1 in DU-145 human prostate cancer cells results in the constitutive activation of JAK1 (15). Therefore, the relatively higher expression levels of p-JAK1 in adjacent non-cancer tissues (Fig. 1 and Table I) may be required for the tumor suppressor activity for certain tumor suppressor genes, such as BRCA1. Besides, a significant correlation between decreased p-JAK1 expression and increased tumor size (Table II) may enforce the speculation that loss or decrease of constitutively activated JAK1 contributes to breast cancer development.

In the present study, an increased p-JAK1 expression in breast cancer tissues was significantly correlated with a negative estrogen receptor status (Table II). The expression of p-JAK1 was decreased in ER-positive breast cancer cells compared to ER-negative breast cancer cells. The relatively lower expression of p-JAK1 in the ER-positive breast cells were reversed by ER $\alpha$-specific siRNA (Fig. 2). Our results suggest that crosstalk exists between p-JAK1 and estrogen receptor in breast cancer contributing to breast cancer development. It has been demonstrated that SHP-1 (SH2 domain-containing protein tyrosine phosphatase-1) inhibits the tyrosine phosphorylation of JAK kinases (16-19). Furthermore, the expression of SHP-1 is low in the ERnegative breast cancer cells (Hs578T, MDA-MB-231 and MDA-MB-436), but normally expressed or overexpressed in the ER positive breast cancer cell lines (MCF-7, BT-20 and ZR-75-1) (20). The modulation of p-JAK1 expression by ER may be in SHP-1 related manner. Alternatively, estrogen receptor may attenuate p-JAK1 expression in a way similar to the observation that estrogen inhibits GH-mediated JAK2 activation by SOCS-2 (11). Although our observations, in vivo and in vitro, demonstrated crosstalk between p-JAK1 and ER in breast cancer, whether this plays a critical role in the development of breast cancer requires further investigation.

In summary, our results indicate that altered expression of p-JAK1 may contribute to breast infiltrating ductal carcinoma, and the modulation of JAK1 expression may be ER-related. Further studies are required to dissect the detailed mechanisms and the potential role of the crosstalk between p-JAK1 and estrogen receptor in breast cancer development.

\section{Acknowledgements}

This study was supported by grants to S.S.Y. (NHRI-EX939306BI and NSC94-2314-B-214-005) and to M.F.H. (NSC93-2314-B-037-082-).

\section{References}

1. Rane SG and Reddy EP: JAKs, STATs and Src kinases in hematopoiesis. Oncogene 21: 3334-3358, 2002.

2. Rane SG and Reddy EP: Janus kinases: components of multiple signaling pathways. Oncogene 19: 5662-5679, 2000.

3. Boudny V and Kovarik J: JAK/STAT signaling pathways and cancer. Janus kinases/signal transducers and activators of transcription. Neoplasma 49: 349-355, 2002.

4. Rodig SJ, Meraz MA, White JM, et al: Disruption of the Jak1 gene demonstrates obligatory and non-redundant roles of the Jaks in cytokine-induced biologic responses. Cell 93: 373-383, 1998.

5. Campbell GS, Yu CL, Jove R and Carter-Su C: Constitutive activation of JAK1 in Src-transformed Cells. J Biol Chem 272: 2591-2594, 1997. 
6. Zhang Y, Turkson J, Carter-Su C, et al: Activation of Stat3 in vSrc-transformed fibroblasts requires cooperation of JAK1 kinase activity. J Biol Chem 275: 24935-2444, 2000.

7. Sexl V, Kovacic B, Piekorz R, et al: Jak1 deficiency leads to enhanced Abelson-induced B-cell tumor formation. Blood 101: 4937-4943, 2003.

8. Welcsh PL, Lee MK, Gonzalez-Hernandez RM, et al: BRCA1 transcriptionally regulates genes involved in breast tumorigenesis. Proc Natl Acad Sci USA 99: 7560-7565, 2002.

9. Winston LA and Hunter T: JAK2, Ras and Raf are required for activation of extracellular signal-regulated kinase/mitogenactivated protein kinase by growth hormone. J Biol Chem 270: 30837-30840, 1995.

10. Xie J, Le Baron MJ, Nevalainen MT and Rui H: Role of tyrosine kinase Jak2 in prolactin-induced differentiation and growth of mammary epithelial cells. J Biol Chem 277: 14020-14030, 2002.

11. Leung KC, Doyle N, Ballesteros M, et al: Estrogen inhibits GH signaling by suppressing GH-induced JAK2 phosphorylation, an effect mediated by SOCS-2. Proc Natl Acad Sci USA 100: 1016-1021, 2003.

12. Proietti C, Salatino M, Rosemblit C, et al: Progestins induce transcriptional activation of signal transducer and activator of transcription 3 (Stat3) via a Jak- and Src-dependent mechanism in breast cancer cells. Mol Cell Biol 25: 4826-4840, 2005.

13. Yuan SS, Cox LA, Dasika GK and Lee EY: Cloning and functional studies of a novel gene aberrantly expressed in RBdeficient embryos. Dev Biol 207: 62-75, 1999.
14. Yuan SS, Tsai KB, Chung YF, et al: Aberrant expression and possible involvement of the leptin receptor in endometrial cancer. Gynecol Oncol 92: 769-775, 2004.

15. Gao B, Shen X, Kunos G, et al: Constitutive activation of JAKSTAT3 signaling by BRCA1 in human prostate cancer cells. FEBS Lett 488: 179-184, 2001.

16. Yi T and Ihle JN: Association of hematopoietic cell phosphatase with c-Kit after stimulation with c-Kit ligand. Mol Cell Biol 13: 3350-3358, 1993.

17. Yi T, Mui AL, Krystal G and Ihle JN: Hematopoietic cell phosphatase associates with the interleukin-3 (IL-3) receptor beta chain and down-regulates IL-3-induced tyrosine phosphorylation and mitogenesis. Mol Cell Biol 13: 7577-7586, 1993.

18. Klingmuller U, Lorenz U, Cantley LC, Neel BG and Lodish HF: Specific recruitment of SH-PTP1 to the erythropoietin receptor causes inactivation of JAK2 and termination of proliferative signals. Cell 80: 729-738, 1995.

19. Bittorf T, Seiler J, Zhang Z, Jaster R and Brock J: SHP-1 protein tyrosine phosphatase negatively modulates erythroid differentiation and suppression of apoptosis in J2E erythroleukemic cells. Biol Chem 380: 1201-1209, 1999.

20. Yip SS, Crew AJ, Gee JM, et al: Up-regulation of the protein tyrosine phosphatase SHP-1 in human breast cancer and correlation with GRB2 expression. Int J Cancer 88: 363-368, 2000 